\title{
Appropriate remeshing for finite element impact analysis with consideration of error and computing efficiency
}

\author{
Toshikazu Torigaki a and Noboru Kikuchi ${ }^{b}$ \\ a Vehicle Research Laboratory, Nissan Research Center, Nissan Motor Co., Ltd., 1 Natsushima-cho, Yokosuka 237, Japan \\ ${ }^{b}$ Department of Mechanical Engineering and Applied Mechanics, Unilersity of Michigan, Ann Arbor, Michigan 48109, USA
}

Received 1 March 1992

\begin{abstract}
Remeshing for finite element impact analysis is discussed to perform an accurate and efficient computation. A nodal relocation scheme is implemented for remeshing based on the following considerations: (1) spatial discretization error of the solution, (2) approximation error of a deformable domain on curved boundaries, (3) stability condition of a time integration scheme. Although the first error is widely known, the second error is new and very important to an impact analysis specially if there exists a large stretching deformation on curved boundaries. To raise the computing efficiency, a stability condition is taken into account. The presented scheme is carefully examined by solving several example problems.
\end{abstract}

\section{Introduction}

The nature of large deformation impact problems of inelastic materials makes error analysis very complicated. A mathematical treatment of these problems is not yet available. For this type of problem, therefore, we have to introduce error measures that are realistic but must also approximate the theory from linear elastic problems to some degree. However, because of nonlinearity and large deformation, extra considerations must be made. Three kinds of errors can be considered: (1) finite element approximation of displacement, (2) discretization in time, and (3) finite element approximation of a domain which undergoes large deformation. The first and the third errors are produced by discretization in space, however, they have totally different characteristics. The first error is similar to that of linear elasticity. If the problem is computed incrementally and the error is analyzed for each increment, derivation of the error measure for displacement in an increment is similar to that of small deformation linear elastic problems. For example, if all formulations are linearized in an increment, the error measure in an increment is equivalent to that of linear

Correspondence to: Dr. Toshikazu Torigaki, Vehicle Research Laboratory, Nissan Research Center, Nissan Motor Co., Ltd., 1 Natsushima-cho, Yokosuka 237, Japan. elastic contact problems. Such an error measure is described in [1]. The second error, due to discretization in time, is called a truncation error, and has been thoroughly studied by many researchers such as [2-6]. The third error due to approximation of a domain which undergoes large deformation, is new to large deformation problems. In small deformation problems, we assume that a domain is completely covered by finite elements and that it is not difficult to generate a finite element mesh without significant error. However, in large deformation problems, elements may undergo large stretching deformation which may not be predictable. If these stretched elements are located on a curved boundary, they will not represent the shape of the original undiscrete smooth domain. Thus, significant integration error is produced due to the difference between the discrete and the undiscrete domains.

If an adaptive mesh refinement is applied to this type of incremental analysis, remapping of all history dependent variables is necessary due to mesh change. Studies have been performed using mesh rezoning in order to avoid the difficulty of continuation of computation due to element crash or severe distortion of a mesh, e.g., by [7-11]. It should be noted that the remapping scheme of variables may produce another kind of error.

In addition to various kinds of error, a stability condition of a numerical time integration scheme is also a very important factor in an adaptive method.

0029-5493/92/\$05.00 (C) 1992 - Elsevier Science Publishers B.V. All rights reserved 
Size of elements and size of time increments are not independent. In general, all factors in the adaptive methods interact with each other. The adaptive method for large deformation impact problems is thus not uniquely defined. It may have different description in different problems. It requires more overall consideration of accuracy in practical computations instead of pursuing precise mathematical analyses, although, if such mathematical studies exist, it is certainly much better to construct a theory on the adaptive method for impact problems.

\section{Adaptive methods in impact problems}

In contrast to the adaptive methods in small deformation problems in which only the spatial discretization error of displacement is considered, more error sources have to be considered for impact problems. We summarize the discussion in the Introduction for impact problems; it is necessary to consider

(1) spatial discretization error of displacement,

(2) truncation error in a time integration scheme,

(3) approximation error of a deformable domain on a curved boundary,

(4) stability condition of a numerical time integration scheme,

(5) remapping error to transfer nodal values both in "old" and "new" meshes.

Contribution of the spatial discretization error of displacement on a contact boundary is not considered since its contribution is not large, see [12]. In an incremental computation of impact problems, the adaptive method may be applied not only to redesign a finite element mesh but also to optimize size of a time increment so that overall error of each increment is equal. However, if an explicit time integration method is used, size of a time increment is already bounded by the stability condition. Its size is determined by a smaller bound of either the truncation error or the stability condition. In general, the bound by the stability condition is much smaller, usually several-hundred to several-thousand times smaller than that of an implicit time integration scheme in which the truncation error is an important factor for solving problems. Therefore, it may not be necessary to consider the truncation error when the adaptive method is concerned. Here the adaptive method is applied only to redesign a finite element mesh. It is noted that the adaptive method can be used to redesign a mesh in order to enlarge unnecessarily small size of a time increment since its size is totally determined by the size of the smallest element in the stability condition. This situation usually occurs when continuous and large compressible loads are applied to a portion of a contact boundary, and the size of time increment sometimes becomes too small to continue computation.

Next question is how many times the adaptive method should be applied during the deformation process. If the adaptive method is applied every time step the remapping error becomes considerably large. It is not possible to reduce all kinds of error or to make all kinds of error equal for all elements in the practical computation, and the engineer's judgment is necessary to determine how many times the adaptive method is applied. We apply the adaptive method ten to forty times during the deformation process so that the deformation in an interval between its applications may satisfy the small deformation assumption and that the remapping error may stay at an inconsiderable level. Note that we do not assume small deformation in the intervals, but that the word "the small deformation assumption" is used to determine when the adaptive method should be applicd.

We summarize purposes of the adaptive methods in impact problems to be that

(1) Redesign a finite element mesh in order to reduce two kinds of error due to spatial discretization of displacement and approximation of a domain on a boundary, or to make them equal for all elements,

(2) Redesign a finite element mesh in order to avoid unnecessarily small size of a time increment.

\section{Error measure}

Let us define two kinds of error measures for the two error sources, which are due to the spatial discretization crror of displacement and due to the domain approximation error on a boundary. An example of the crror measure for the first error source has been given by [1] in which the error measure is defined using the energy norm of the interpolation error in an increment. However, in many engineering applications, the most important issue of impact problems is how deformation occurs or how destruction occurs. A major factor of this aspect is a plastic strain for most of metallic materials. The strain energy may not show the deformation faithfully since a large amount of energy may be being dissipated in a plastic process. Thus the error measure $E_{c}$ for the first error source may be defined by

$E_{\mathrm{e}}=\left\{\int_{\Omega_{\mathrm{c}}}\left[\bar{\epsilon}^{\mathrm{p}}\left(\boldsymbol{u}-\boldsymbol{w}_{\mathrm{h}}\right)\right]^{2} \mathrm{~d} \Omega\right\}^{1 / 2}$, 
where $\bar{\epsilon}^{\mathrm{p}}$ denotes the effective plastic strain, $\boldsymbol{u}$ is the true displacement and $w_{h}$ is its interpolation. Note that the interpolation error well represents the discretization error, see [12] for justification. Although there are more accurate ways to calculate the discretization error other than using the interpolation error, see [12,13], we shall apply the interpolation approach in the present method since the interpolation error can provide good error estimates using much shorter computing time than the other ways, and since reduction of computing time is very important in a large scale computation such as impact problems. Eq. (1) measures the accumulated error from the initial state. Letting $\Delta$ denote an interval between applications of the adaptive method, the error measure which measures error in the intervals may also be defined by

$\Delta E_{\mathrm{e}}=\left\{\int_{\Omega_{\mathrm{c}}}\left[\Delta \bar{\epsilon}^{\mathrm{p}}\left(\boldsymbol{u}-\boldsymbol{w}_{\mathrm{h}}\right)\right]^{2} \mathrm{~d} \Omega\right\}^{1 / 2}$.

Usage of (1) and (2) is discussed later.

The domain approximation error is caused by significant stretching of elements which are located on a curved boundary. Letting $d_{\mathrm{h}}$ be the normal distance between a discrete and an undiscrete domain on a boundary and using the effective plastic strain $\bar{\epsilon}^{\mathrm{p}}$ as a principal value in impact analyses similarly to (1), the error measure for the domain error may be defined by

$E_{\mathrm{e}}=\left\{\int_{\partial \Omega_{\mathrm{e}} \cap \Gamma} d_{\mathrm{h}}\left[\bar{\epsilon}^{\mathrm{p}}(u)\right]^{2} \mathrm{~d} \partial \Omega\right\}^{1 / 2}$.

Let $s$ be the parametric coordinates of a surface element. If the domain is discretized by QUAD4 or HEXA8 elements *, the surface element is a LINE2 or a QUAD4 element respectively, and $-1 \leq s_{1} \leq 1$, $i=1$ for the LINE2 or $i=1,2$ for the QUAD4 element. Then the normal distance $d_{\mathrm{h}}$ may be approximated by

$d_{\mathrm{h}}(s) \approx\left|x(s)-x_{\mathrm{h}}(s)\right|$,

where $x_{\mathrm{h}}(s)$ and $x(s)$ denote coordinates of the discrete boundary and its smoothed version, which have the relations:

$x(s)-x_{\mathrm{h}}(s)=\frac{1}{2} \frac{\partial^{2} x(s)}{\partial s_{1}^{2}}\left(s_{1}^{2}-1\right)$

* QUAD4 and HEXA8 do not mean the special element type available in MSC-NASTRAN. They merely indicate four node guadrilateral and eight node hexahedral elements, respectively. for the LINE2 element, and

$$
\begin{aligned}
x(s)-x_{\mathrm{h}}(s)= & \frac{1}{2} \frac{\partial^{2} x(s)}{\partial s_{1}^{2}}\left(s_{1}^{2}-1\right)+\frac{1}{2} \frac{\partial^{2} x(s)}{\partial s_{2}^{2}}\left(s_{2}^{2}-1\right) \\
& -\frac{1}{4} \frac{\partial^{4} x(s)}{\partial s_{1}^{2} \partial s_{2}^{2}}\left(s_{1}^{2}-1\right)\left(s_{2}^{2}-1\right)
\end{aligned}
$$

for the QUAD4 element. The smoothed boundary $x(s)$ can be obtained using the weighted averaging scheme, see [12] for details. The error measure in the intervals may also be defined by

$$
\begin{aligned}
\Delta E_{\mathrm{e}}= & \left\{\int_{\partial \Omega_{\mathrm{e}} \cap \Gamma} \Delta d_{\mathrm{h}}\left[\bar{\epsilon}^{\mathrm{p}^{*}}(u)\right]^{2} \mathrm{~d} \partial \Omega\right. \\
& \left.+\int_{\partial \Omega_{\mathrm{v}} \cap \Gamma} d_{\mathrm{h}}^{*}\left[\Delta \overline{\boldsymbol{\epsilon}}^{\mathrm{p}}(u)\right]^{2} \mathrm{~d} \partial \Omega\right\}^{1 / 2},
\end{aligned}
$$

where ${ }^{*}$ in $\bar{\epsilon}^{\mathrm{p}^{*}}$ and $d_{\mathrm{h}}^{*}$ denotes values obtained at the midpoint of the intervals.

Combining (1) and (3), and (2) and (7), the error measures which include both error sources may be defined by

$$
\begin{aligned}
E_{\mathrm{e}}= & \left\{\int_{\Omega_{\mathrm{e}}}\left[\bar{\epsilon}^{\mathrm{p}}\left(u-w_{\mathrm{h}}\right)\right]^{2} \mathrm{~d} \Omega\right. \\
& \left.+\int_{\partial \Omega_{\mathrm{e}} \cap \Gamma} d_{\mathrm{h}}\left[\bar{\epsilon}^{\mathrm{p}}(u)\right]^{2} \mathrm{~d} \partial \Omega\right\}^{1 / 2}
\end{aligned}
$$

and

$$
\begin{aligned}
\Delta E_{\mathrm{e}}= & \left\{\int_{\Omega_{\mathrm{e}}}\left[\Delta \bar{\epsilon}^{\mathrm{p}}\left(u-w_{\mathrm{h}}\right)\right]^{2} \mathrm{~d} \Omega\right. \\
& +\int_{\partial \Omega_{\mathrm{c}} \cap \Gamma} \Delta d_{\mathrm{h}}\left[\bar{\epsilon}^{\mathrm{p}^{*}}(u)\right]^{2} \mathrm{~d} \partial \Omega \\
& \left.+\int_{\partial \Omega_{\mathrm{c}} \cap \Gamma} d_{\mathrm{h}}^{*}\left[\Delta \bar{\epsilon}^{\mathrm{p}}(u)\right]^{2} \mathrm{~d} \partial \Omega\right\}^{1 / 2} .
\end{aligned}
$$

It is noted that the error measures (3) and (7) consist of boundary integral terms, and then (8) and (9) include both domain and boundary integral terms. This leads to a difficulty for the $r$-adaptive method. The boundary integral terms can sometimes become very large even though the area of an element becomes almost zero, and these terms still make the element smaller. This may result to such a small size of the time increment that continuation of the computation becomes impossible. Therefore, (8) and (9) should not be used for the $r$-method. Another error measure for the 
domain approximation error needs be defined to avoid such a difficulty. To do this, it is noted that the domain approximation error is caused by significant stretching of elements. Thus, the effective plastic strain can represent the magnitude of such stretching. Although the domain approximation should be confined in the neighborhood of the boundary of the domain, we regard that its error is generated by the accumulation of stretching of all the elements of the domain. We, however, have to admit that this statement is not exactly correct in all the possible deformations, but it can have a co-relation to the amount of domain approximation error. Thus, we define

$E_{\mathrm{e}}=\left\{\int_{\Omega_{\mathrm{c}}}\left[\bar{\epsilon}^{\mathrm{p}}(\boldsymbol{u})\right]^{2} \mathrm{~d} \Omega\right\}^{1 / 2}$,

and

$\Delta E_{\mathrm{e}}=\left\{\int_{\Omega_{\mathrm{e}}}\left[\Delta \bar{\epsilon}^{\mathrm{p}}(u)\right]^{2} \mathrm{~d} \Omega\right\}^{1 / 2}$,

which are the error measures related to the domain approximation error. The effective plastic strain represents not only stretching deformation but also all other types of deformation except that by hydrostatic loading condition.

With (10) and (11), the error measures for combined error sources may be defined by

$$
\begin{aligned}
E_{\mathrm{e}}= & \left\{\left[\int_{\Omega_{\mathrm{c}}}\left[\overline{\boldsymbol{\epsilon}}^{\mathrm{p}}\left(\boldsymbol{u}-\boldsymbol{w}_{\mathrm{h}}\right)\right]^{2} \mathrm{~d} \Omega\right]^{m / 2}\right. \\
& \left.\times\left[\int_{\Omega_{\mathrm{c}}}\left[\overline{\boldsymbol{\epsilon}}^{\mathrm{p}}(u)\right]^{2} \mathrm{~d} \Omega\right]^{n / 2}\right\}^{1 /(m+n)}
\end{aligned}
$$

and

$$
\begin{aligned}
\Delta E_{\mathrm{e}}= & \left\{\left[\int_{\Omega_{\mathrm{c}}}\left[\Delta \overline{\boldsymbol{\epsilon}}^{\mathrm{p}}\left(\boldsymbol{u}-\boldsymbol{w}_{\mathrm{h}}\right)\right]^{2} \mathrm{~d} \Omega\right]^{m / 2}\right. \\
& \left.\times\left[\int_{\Omega_{\mathrm{c}}}\left[\Delta \overline{\boldsymbol{\epsilon}}^{\mathrm{P}}(\boldsymbol{u})\right]^{2} \mathrm{~d} \Omega\right]^{n / 2}\right\}^{1 /(m+n)},
\end{aligned}
$$

where $m$ and $n$ denote weights for two error sources. Additive composition is not allowed here since two kinds of the error measures imply different quantities.

\section{Simplified computation of interpolation error}

Computation of the error measure which includes the interpolation error $\boldsymbol{u}-\boldsymbol{w}_{\mathrm{h}}$ is time consuming, for example, if the crror measure consists of $\bar{\epsilon}^{\mathrm{P}}\left(\boldsymbol{u}-\boldsymbol{w}_{\mathrm{h}}\right)$, it is required to be updated in every time steps as well as $\bar{\epsilon}^{\mathrm{p}}$, and such computation usually spends nearly half of the total computing time. On the other hand, the error measure is needed only when the adaptive method is applied, that is, every hundreds or every thousands of time steps in the explicit time integration scheme. Thus, if $\overline{\boldsymbol{\epsilon}}^{\mathrm{p}}\left(\boldsymbol{u}-\boldsymbol{w}_{\mathrm{h}}\right)$ is approximately computed by using $\bar{\epsilon}^{\mathrm{p}}$ only when it is needed, computation of the error measure itself may be trivial in the whole computation.

Letting $q$ be a principal property for the error measure $E_{\mathrm{e}}$ such as $\bar{\epsilon}^{\mathrm{p}}, E_{\mathrm{e}}$ duc to the interpolation error is given by

$E_{\mathrm{c}}=\left\{\int_{\Omega_{\mathrm{c}}}\left[q\left(\boldsymbol{u}-\boldsymbol{w}_{\mathrm{h}}\right)\right]^{2} \mathrm{~d} \Omega\right\}^{1 / 2}$,

and may be approximated by

$E_{\mathrm{e}} \approx\left\{\int_{\Omega_{\mathrm{i}}}\left[q(u)-q\left(\boldsymbol{w}_{\mathrm{h}}\right)\right]^{2} \mathrm{~d} \Omega\right\}^{1 / 2}$.

This approximation is exact if $q$ is a linear function of $\boldsymbol{u}-\boldsymbol{w}_{\mathrm{h}}$, and such condition is approximately satisfied for small deformation linear-elastic problems for the principal property $q$ such as von Mises equivalent stress. Now, let us reconstruct a one-higher-order function $q^{*}$ than $q\left(\boldsymbol{u}_{\mathrm{h}}\right)$ so that $q^{*}-q\left(\boldsymbol{u}_{\mathrm{h}}\right)$ may become a proper approximation of $q(u)-q\left(w_{\mathrm{h}}\right)$ where $u_{\mathrm{h}}$ is the displacement obtained by a finite element method. Such a function can be obtained by a weighted averaging scheme. If $q\left(u_{h}\right)$ is constant in each element, $q^{*}$ is obtained as a piecewise continuous bilinear function or a piecewise continuous trilinear function for the domain consisting of QUAD4 or HEXA8 elements, respectively. It is noted that, if $\bar{\epsilon}^{\mathrm{p}}$ is taken to be $q, q\left(\boldsymbol{u}_{\mathrm{h}}\right)$ is constant in each element in the case that the full reduced integration rule (one point integration) is applied to construct the internal load vector. The order of the function is defined such that piecewise constant $\rightarrow$ piecewise continuous bilinear $\rightarrow$ piecewise continuous biquadratic for the domain consists of QUAD4 clements, and that piecewise constant $\rightarrow$ piecewise continuous trilinear $\rightarrow$ piecewise continuous triquadratic for the domain consists of HEXA8 elements. The one-higher-order function means a function which can be properly reconstructed from the original function by the weighted averaging scheme; see [12] for details. However, in mathematics, there are many cases that $\epsilon$ can not be described by a continuous function which is bounded, in which a weighted averaging scheme does not work well. Treatment of such cases are also discussed in [12]. 
Letting $q^{*}$ which is evaluated at the center of an element that is the same value of constant $q\left(u_{\mathrm{h}}\right),(15)$ for a QUAD4 element can be approximated by

$$
\begin{gathered}
E_{\mathrm{e}}=\left\{\int _ { \Omega _ { \mathrm { e } } } \left[\left(\partial q^{*} / \partial s_{1}\right) s_{1}+\left(\partial q^{*} / \partial s_{2}\right) s_{2}\right.\right. \\
\left.\left.+\left(\partial^{2} q^{*} / \partial s_{1} \partial s_{2}\right) s_{1} s_{2}\right]^{2} \mathrm{~d} \Omega\right\}^{1 / 2},
\end{gathered}
$$

where $s$ denotes the parametric coordinates and all derivatives of $q^{*}$ with respect to the parametric coordinates are evaluated at $\boldsymbol{s}=\mathbf{0}$. If further assumptions are made, in which an element is almost a parallelogram (i.e., the determinant of the Jacobian matrix is almost constant in an element), (16) can be explicitly written as

$$
E_{\mathrm{e}}=\left\{\frac{4 J}{3}\left(\frac{\partial q^{*}}{\partial s_{1}}\right)^{2}+\frac{4 J}{3}\left(\frac{\partial q^{*}}{\partial s_{2}}\right)+\frac{4 J}{9}\left(\frac{\partial^{2} q^{*}}{\partial s_{1} \partial s_{2}}\right)^{2}\right\}^{1 / 2},
$$

where $J$ is the determinant of the Jacobian matrix at the center of the element. A similar explicit expression of $E_{\mathrm{e}}$ for HEXA8 elements is also obtained as

$$
\begin{aligned}
E_{\mathrm{e}}= & \left\{\frac{8 J}{3}\left(\frac{\partial q^{*}}{\partial s_{1}}\right)^{2}+\frac{8 J}{3}\left(\frac{\partial q^{*}}{\partial s_{2}}\right)^{2}+\frac{8 J}{3}\left(\frac{\partial q^{*}}{\partial s_{3}}\right)^{2}\right. \\
& +\frac{8 J}{9}\left(\frac{\partial^{2} q^{*}}{\partial s_{1} \partial s_{2}}\right)^{2}+\frac{8 J}{9}\left(\frac{\partial^{2} q^{*}}{\partial s_{2} \partial s_{3}}\right)^{2} \\
& \left.+\frac{8 J}{9}\left(\frac{\partial^{2} q^{*}}{\partial s_{3} \partial s_{1}}\right)^{2}+\frac{8 J}{27}\left(\frac{\partial^{3} q^{*}}{\partial s_{1} \partial s_{2} \partial s_{3}}\right)^{2}\right\}^{1 / 2} .
\end{aligned}
$$

Using the small deformation linear-elastic problem which is shown in fig. 1, two kinds of the error measures (14) and (15) are computed and compared. The von Mises equivalent stress is taken as $q$ in this example. Figure 2 shows the error measures along the lines $A-A^{\prime}$ and $B-B^{\prime}$ shown in fig. 1 . As the result shows,

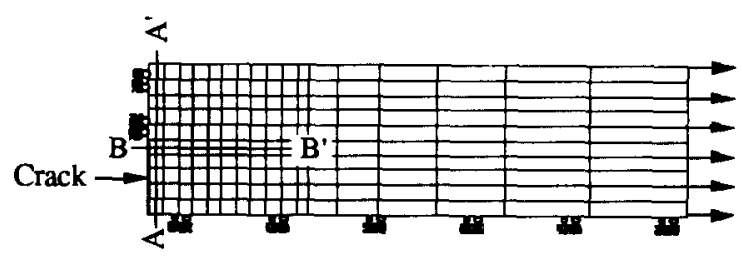

Fig. 1. Finite element model.

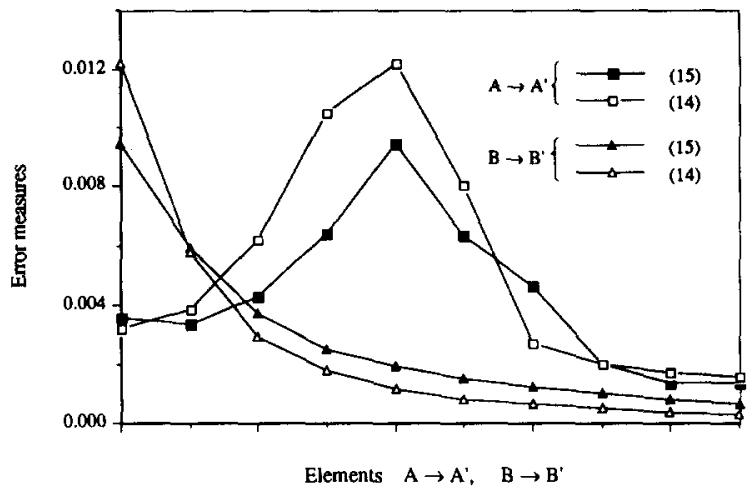

Fig. 2. Comparison of the error measures (14) and (15) by taking $q=\bar{\sigma}$.

the error measure (15) is less sensitive in elements with higher gradient of $q$. The gradient of $q$ becomes infinite at the crack tip. However, in impact problems where $\bar{\epsilon}^{\mathrm{p}}$ is taken as $q, q$ is much more smoothly distributed. Thus, (15) is used for our numerical examples since it is the only error measure which is practical and can be computed with a lot smaller amount of computing time.

\section{Mesh design in impact problems}

The adaptive mesh optimization is essentially the same as that of linear elastic problems. In impact problems, it is very difficult to design the optimal mesh in the overall sense because there are too many error sources. In this paper, two purposes have been specified and several kinds of error measures have been defined. Here, we shall discuss a mesh design method and examine the error measures introduced in the previous section by showing several test examples rather than pursuing precise mathematical analyses. Only the $r$-method is used simply because of restriction of computing time. The $h$-method makes the size of the time increments too small and it is not realistic in this type of problems.

There are two new subjects specially for impact problems. Following [12], the post-projection scheme is used to relocate nodes on a boundary. In contrast to small deformation problems, the boundary shape is not prescribed and must be obtained by using the existing finite element mesh. If the finite element mesh itself is used to describe the boundary shape, repeated relocation of boundary nodes produces significant shape change since discrete boundary shape may be given by 
a piecewise linear or a piecewise bilincar function. Then some procedure to estimate the undiscrete boundary shape is necessary. One of such procedures is given in (5) and (6). All boundary nodes are projected onto the estimated undiscrete boundary after relocation, see [12] for details. The other subject is a remapping scheme of all history dependent variables due to the mesh change. This subject is discussed later.

Now, let us show two test examples. The first one is solved to examine the effect of various error measures (1), (2) and (10)-(13) defined in the previous section. In the adaptive method in incremental analysis, we do not repeat the analysis to obtain an adapted mesh as in stationary problems, but an adapted mesh is generated for future time increments using the error measure obtained at the current state. An immediate question is which error measure is better suited for this purpose. More precisely, is a measure of accumulated error from the initial state better than a measure of error in the current interval. Here, the interval means that defined between the previous application of the adaptive method and the current state. The adaptive method is applied so that the deformation in the interval may be small enough to satisfy the small deformation assumption but, as we mentioned, it is not applied at each time increment.

Using the simulation problem of necking in a simple tension test, the crror measures are compared. The finite element model and its dimensions are shown in fig. 3 where axisymmetry is assumed. A nuclear-pressure-vessel steel is used and its material propertics are shown in fig. 4 . Letting $L_{0}$ and $L$ be the initial and the current length of the model, the simulation is stopped at $L / L_{0}=1.28$. Since deformation in early stages of the simulation is just uniform stretch, the adaptive method is not applied in this stage, but it is applied twelve times between $L / L_{0}=1.14$ and $L / L_{0}=1.28$. It is noted that the visible necking starts at $L / L_{0}=$ 1.14. The finite element mesh and the effective plastic strain at $L / L_{0}=1.28$ are shown in figs. $5-10$ where the error measures (1), (2) and (10)-(13) are used respectively. For (12) and (13), $m=n=1$ are chosen. To compare with the results of the unadapted mesh, its

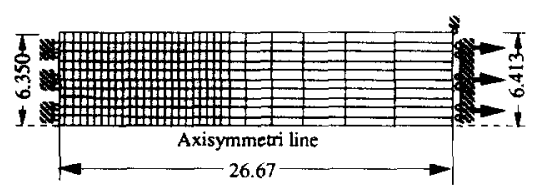

Fig. 3. Necking simulation in a simple tension test, initial setting.

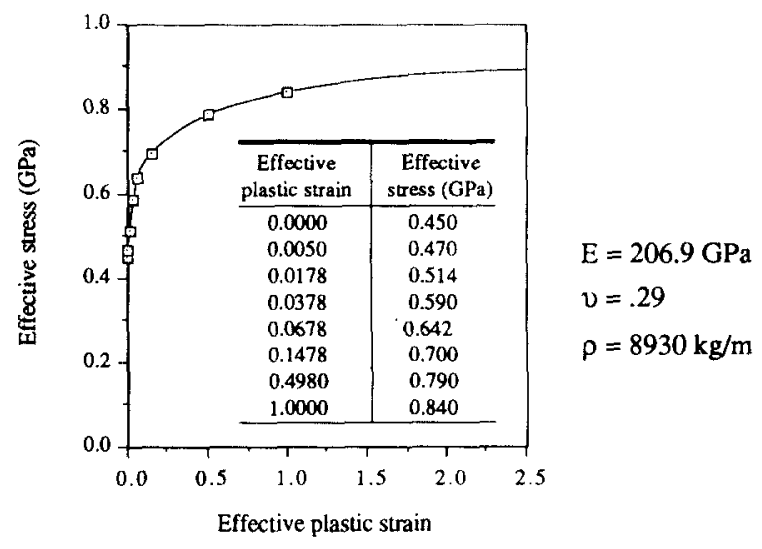

Fig. 4. Material properties of the test specimen.

results are shown at the left side of the adapted ones in all figures. Table 1 compares diameter at the neck, the maximum effective plastic strain and total time steps used in the computation.

As the results show, if the accumulated error measure is applied, the highest value of the effective plastic strain $\bar{\epsilon}^{\mathrm{p}}$ is computed to be lower than the one by the error measure in an interval. Since at the necking portion, the effective plastic strain is expected to be considerably large, the error measure in an interval seems to be better than the accumulated one in this example. The shape of necking has a kink at the center if the discretization error based on the interpolation is applied, i.e., less smooth necking is obtained, while the domain approximation error provides less kinked much smoother shapes of necking both in the accumulated and each individual interval error measures. However, the maximum value of the effective plastic strain is evaluated to be lower than in the case of discretization crror. In this sense, the combined scheme of the discretization and domain approximation errors in each interval (13) seems to be the best choice in this particular example.

Now we shall pay more attention to a mesh design method for impact problems. The error measure is not used in the next examples in which the $r$-adaptive method is applied to avoid unnecessarily small size of the time increments. In other words, the r-adaptive method is applied to remodel the updated finite element mesh by progress of deformation so that very deformed elements are reshaped into much more regular elements. Thus, the main purpose of this study is not the reduction of the approximation error as in usual problems for the adaptive method, but is the 

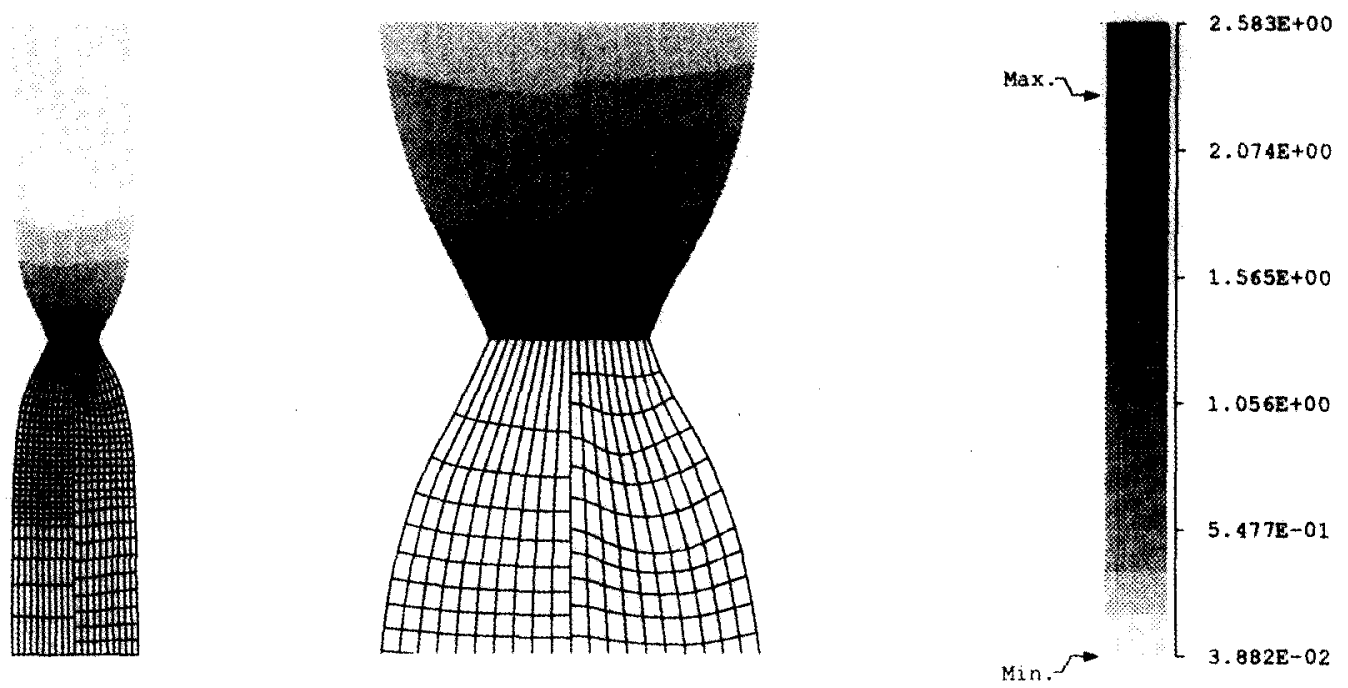

Fig. 5. Final shape and effective plastic strain, adapted by (1) (Discretization/Accumulated).

relocation of the mesh to introduce regular size elements. Since the size of the time increment is dominated by the smallest size of the elements, it can be considerably enlarged without reducing much of accuracy. The rod impact problem, see fig. 11 and fig. 12 for physical setting and material properties of the rod, is solved. In this example, a large compressible load is continuously applied to elements at the bottom part of the rod, and the size of the time increment becomes smaller and smaller because of accumulated compressible deformation over there. To avoid this, the $r$-adaptive method is applied with error measure which is identified with the area of elements to equalize the area of all elements and to enlarge the size of the time
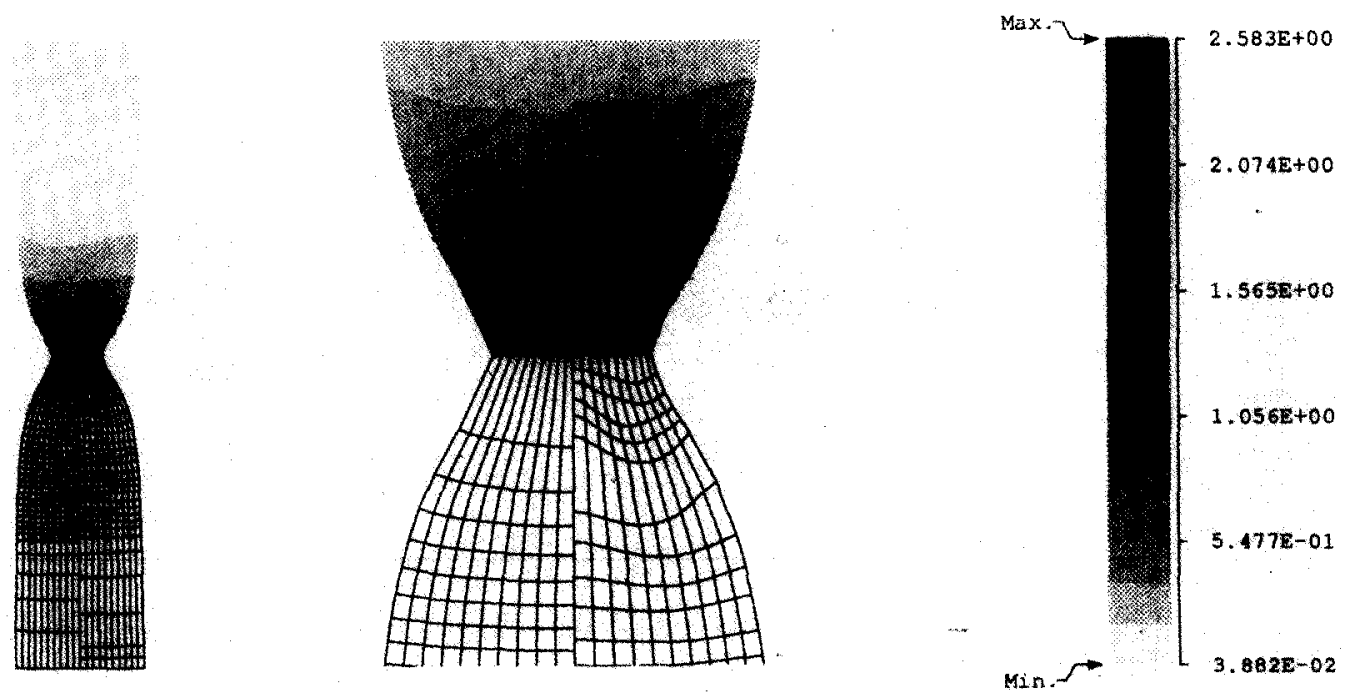

Fig. 6. Final shape and effective plastic strain, adapted by (2) (Discretization/Interval). 

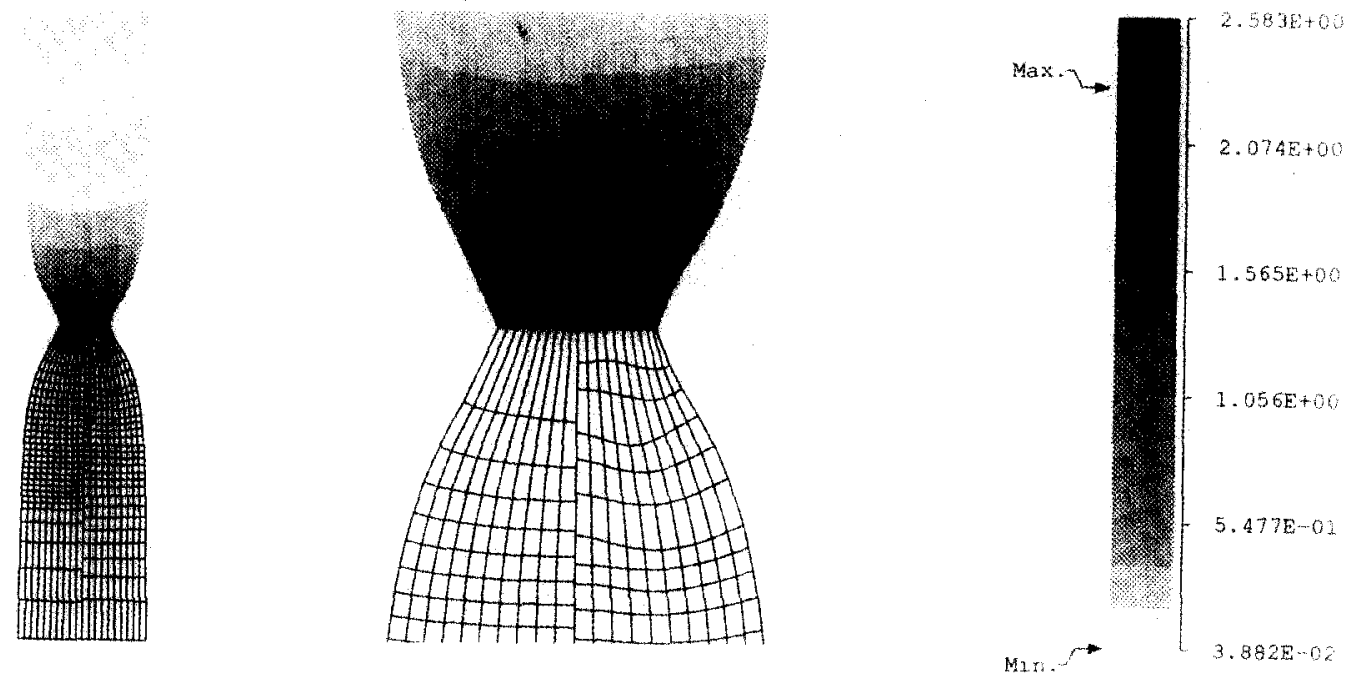

Fig. 7. Final shape and effective plastic strain, adapted by (10) (Domain/Accumulated).

increment. Two finite element meshes are made with 250 and 1000 elements initially. The adaptive method is applied when the current size of the time increment becomes smaller than $70 \%$ of that at the beginning of the current interval. The shape and distribution of the effective plastic strain at $80 \mu \mathrm{s}$ after the impact are shown in fig. 13 and fig. 14 for 250 and 1000 elements meshes respectively. To compare with the results of the unadapted meshes, unadapted results are shown at the left side, while adapted ones are given at the right side
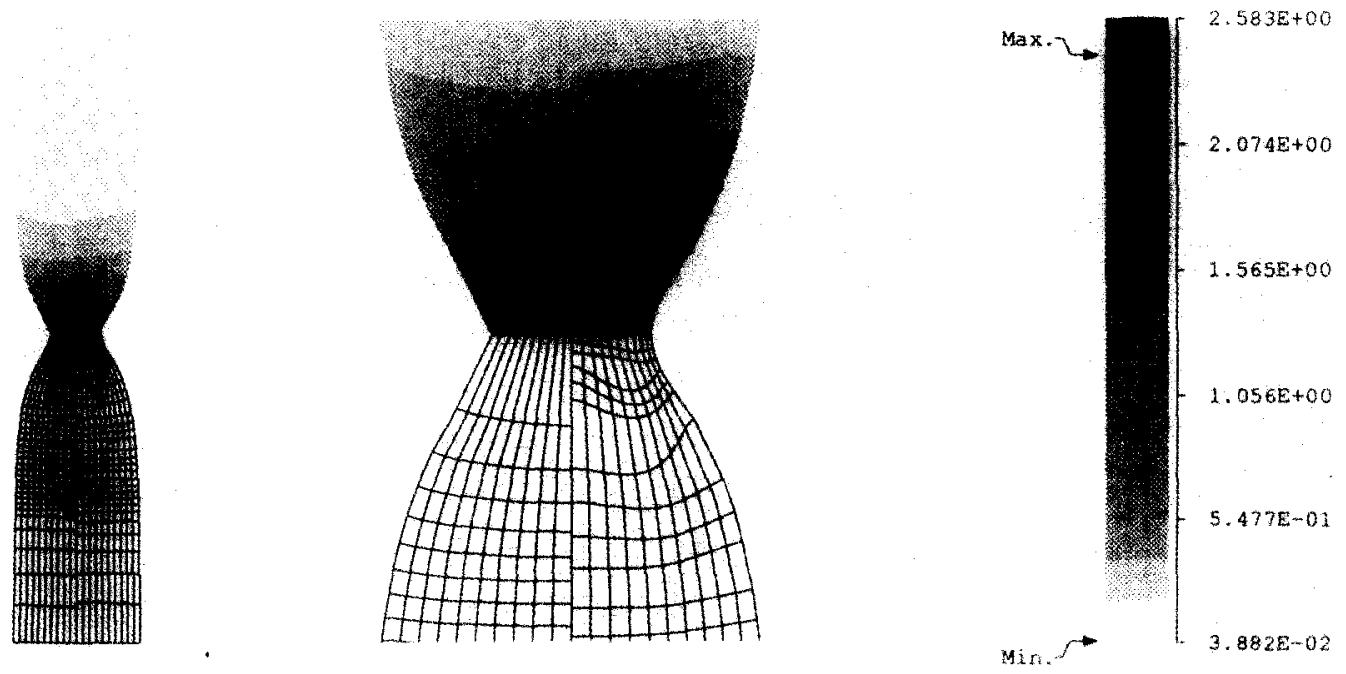

Fig. 8. Final shape and effective plastic strain, adapted by (11) (Domain/Interval). 

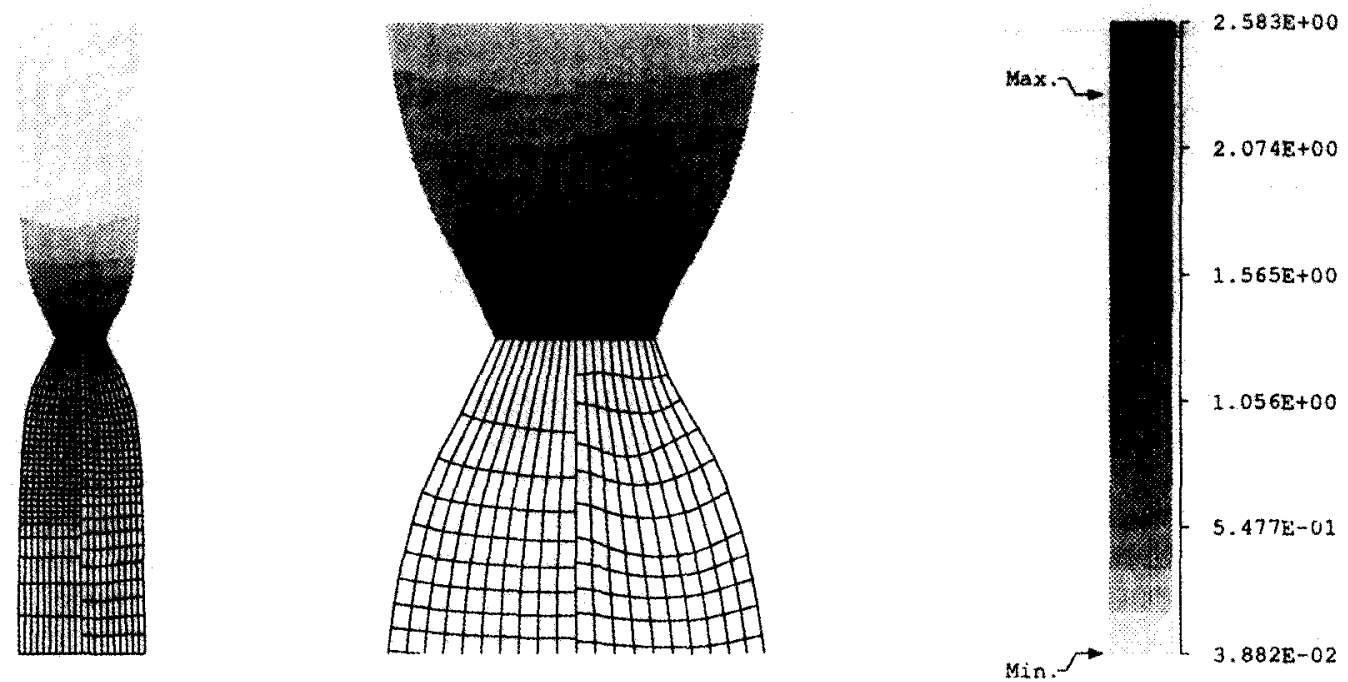

Fig. 9. Final shape and effective plastic strain, adapted by (12) (Discretization-Domain/Accumulated).
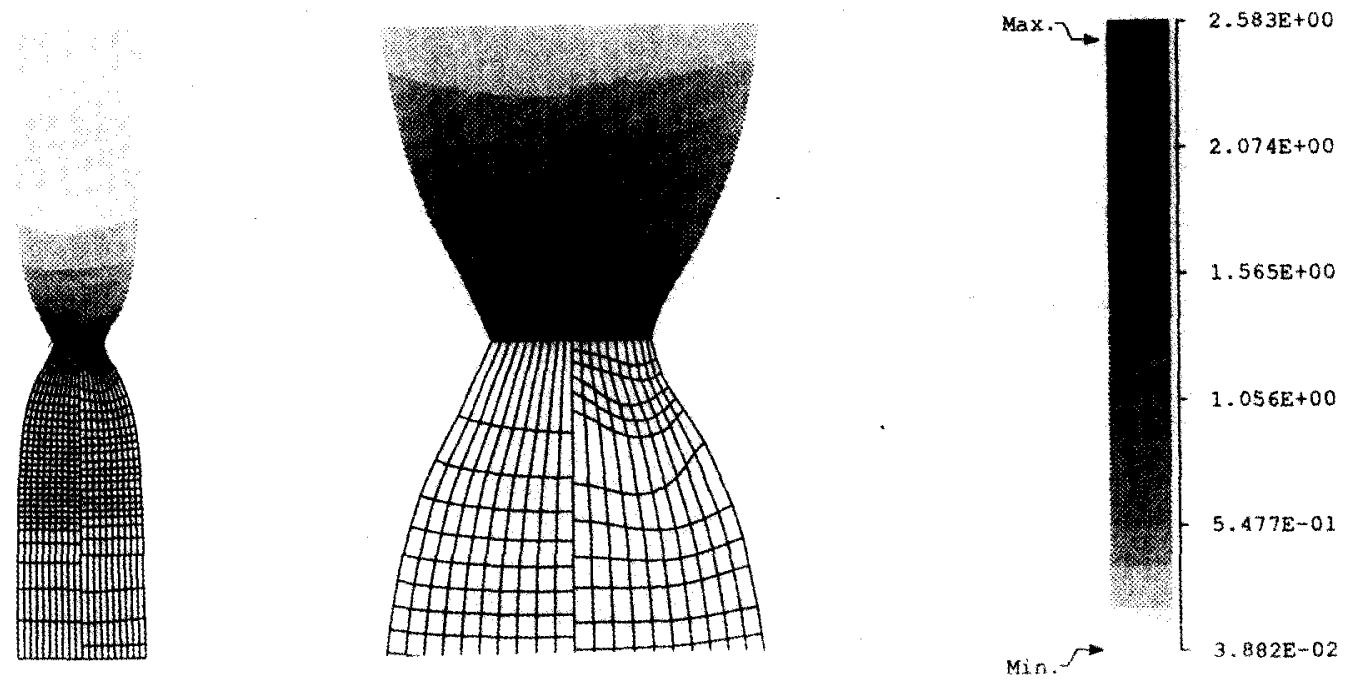

Fig. 10. Final shape and effective plastic strain, adapted by (13) (Discretization-Domain/Interval).

Table 1

Neck diameter, maximum effective plastic strain and total time steps used in the computation

\begin{tabular}{lcccccccc}
\hline Model & Initial & \multicolumn{2}{c}{ R-adapted } & & & & \\
Error measures & & $(1)$ & $(2)$ & $(11)$ & $(12)$ & $(13)$ & $(14)$ \\
\hline Neck diameter (mm) & 4.60 & 4.62 & 4.60 & 4.58 & 4.60 & 4.60 & 4.60 \\
Max. $\bar{\epsilon}^{p}$ & 1.87 & 2.29 & 2.58 & 2.30 & 2.44 & 2.29 & 2.51 \\
Total time steps & 23985 & 24852 & 26144 & 25037 & 26949 & 24700 & 26377 \\
\hline
\end{tabular}




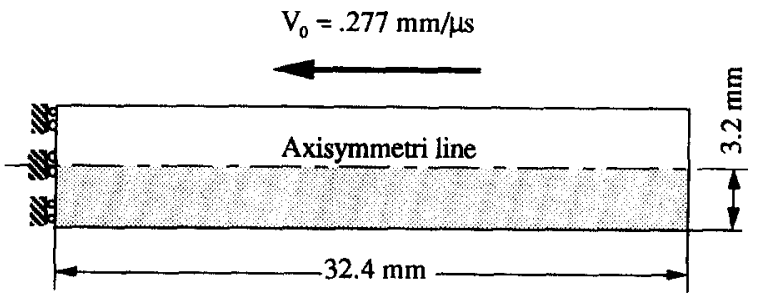

Fig. 11. Rod impact problem, initial setting.

in all figures. Table 2 compares height and bottom diameter of the rod as well as the total time steps used in the computation.

As shown in table 2, reduction of the total time steps is significant; only $21.2 \%$ of the unadapted mesh is required for a 250 element mesh, while only $18.8 \%$ of the unadapted mesh is required for a 1000 element mesh. It is also noted that there is almost no visible difference between adapted and unadapted meshes in

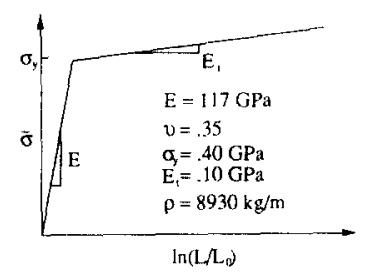

Fig. 12. Material properties of the impacted rod.

deformation and in distribution of the effective plastic strain. This example shows that the adaptive method based on the approximation error is not very critical since there is no singular behavior in the given problem. A uniform finite element mesh with sufficient amount of degrees of freedom can provide sufficiently accurate results. But the issue in this problem is how much reduction of computing time is possible without decreasing accuracy of approximation by applying the
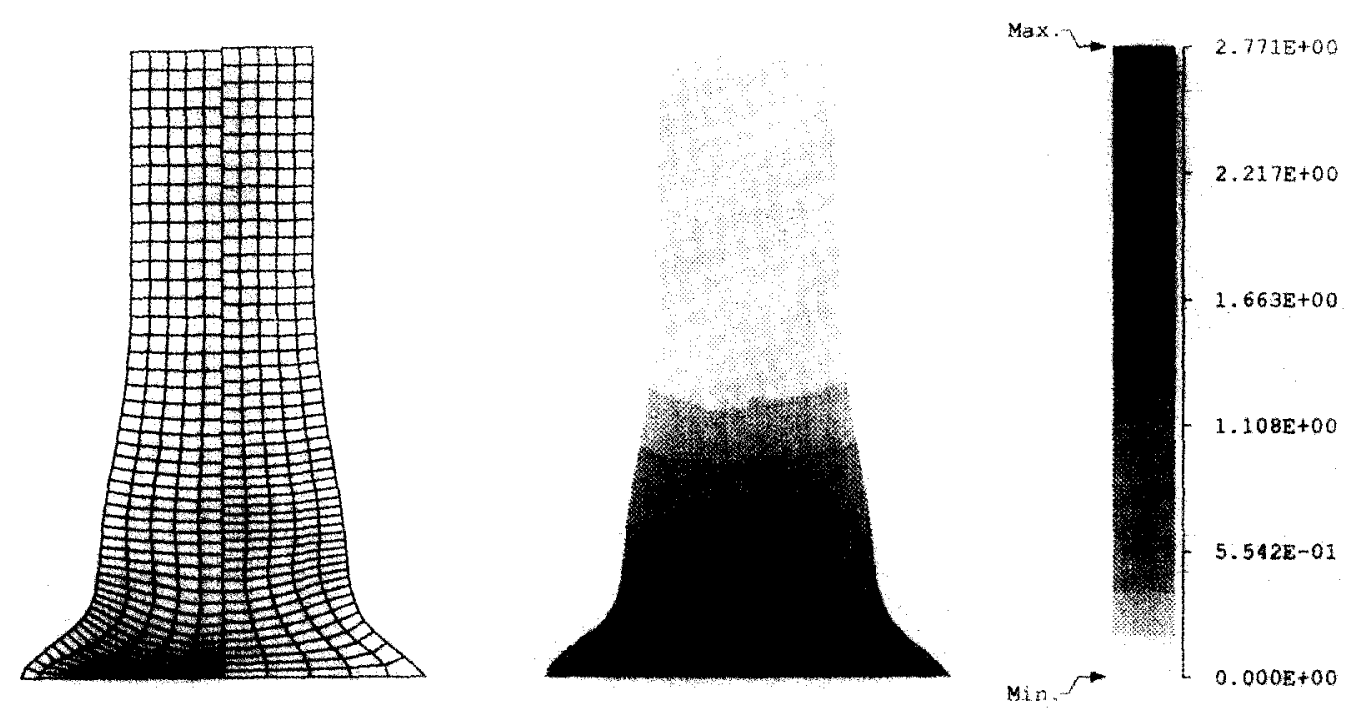

Fig. 13. Final shape and effective plastic strain, 250-element mesh.

Table 2

Height, bottom diameter and total time steps used in computation.

\begin{tabular}{|c|c|c|c|c|}
\hline \multirow{2}{*}{$\begin{array}{l}\text { Model } \\
\text { Number of elements }\end{array}$} & \multicolumn{2}{|l|}{ Initial } & \multicolumn{2}{|c|}{ R-adapted } \\
\hline & 250 & 1000 & 250 & 1000 \\
\hline Height $(\mathrm{mm})$ & 21.43 & 21.42 & 21.53 & 21.48 \\
\hline Bottom diameter $(\mathrm{mm})$ & 14.2 & 14.3 & 14.3 & 14.1 \\
\hline Total time steps & 12631 & 29724 & 2673 & 5586 \\
\hline
\end{tabular}


concept of the $r$-adaptive method. It is clear that it can be applicable as an automatic rezoning (remeshing) scheme which yields a more regular and uniform finite element mesh.

It may be worthwhile to note that the adaptive method with the approximation error is powerful to reduce the error when some sort of singular behavior is expected by existence of cracks, interfaces of dissimilar materials, point loads, shock wave fronts, etc., while it may be less useful if the singular behavior does not exist. If deformation is localized without rapid change of stress/strain in a certain portion of the domain, the $r$-adaptive method with an artificial error measure such as the area of elements may be useful to reduce the overall effort to simulate such a problem.

\section{Remapping}

Because of mesh modification by the adaptive method, the value of all history dependent variables must be remapped to the new location of nodal points or integration points. The variables defined at nodal points, such as the acceleration and the displacement, can be easily remapped using the shape functions of an element such that

$q(s)=q_{\alpha} \xi_{\alpha}(s)$,

where $q(s)$ is the value at the new location of nodal points or integration points, $q_{\alpha}$ is the value at the $\alpha$-th node (in local numbering) of the element to which the new point belongs and $s$ is the position of the new point in the parametric coordinate system. However, for the variables defined at integration points, such as the stress, the radius of the yield function and the back stress, it is necessary to obtain their nodal values before using the shape functions. The least squares methods is the standard to obtain such nodal values, see [10], [11]. Letting $\bar{q}$ be the value at an integration point, i.e., the center of an element, and letting $\bar{q}$ be considered as a constant in each element in our numerical scheme, the least squares method is defined for any function $q$ by

$$
\operatorname{Min}_{q} \frac{1}{2} \int_{\Omega}(q-\bar{q})^{2} \mathrm{~d} \Omega .
$$

If $q=q_{\alpha} \xi_{\alpha}$ and $q$ is continuous, (20) gives a unique value $q_{\mathrm{n}}$ for all nodal points with the least error. The least squares method can provide sufficiently accurate results only for a uniform regular mesh, see [12]. In other words, this method always pursues the best for the whole system and sometimes ignores the best for individual elements. If this method is applied many times for a non-regular finite element mesh, the error in individual elements becomes sufficiently large, and then an oscillatory least squares result of $q$ is obtained. Thus, repeated application of the least squares method must be avoided.
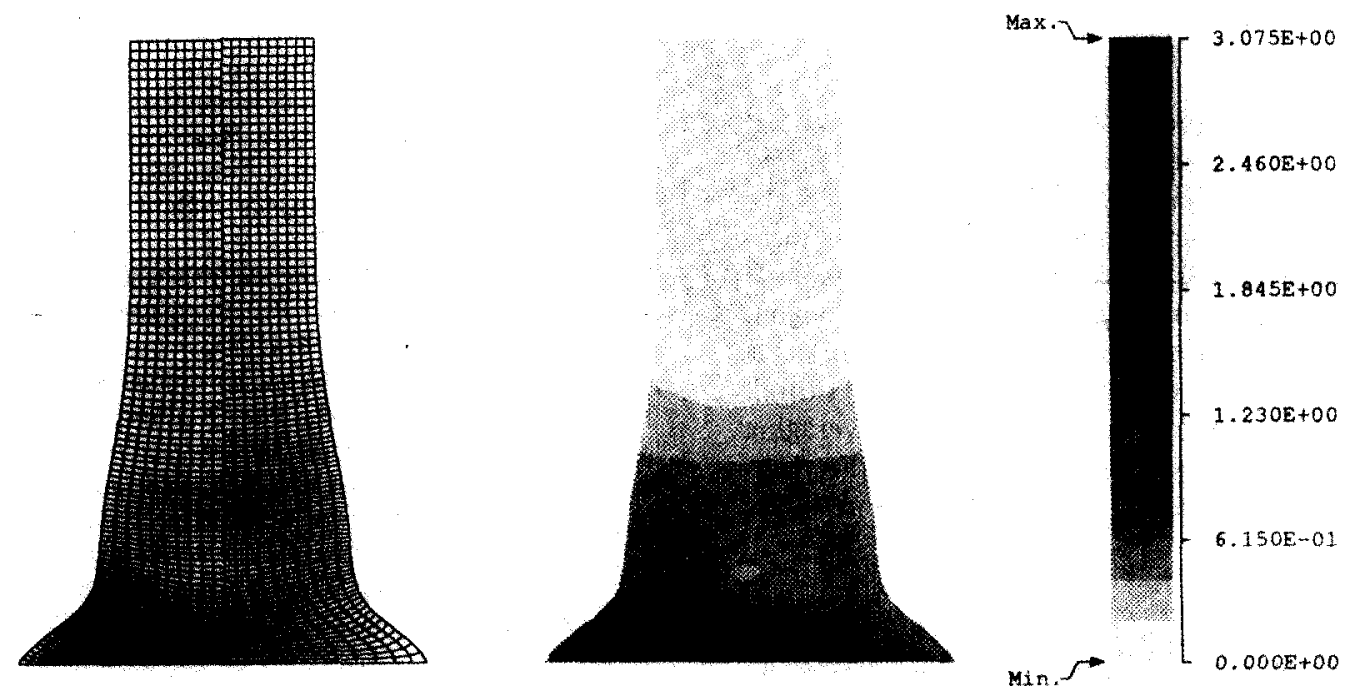

Fig. 14. Final shape and effective plastic strain, 1000-element mesh. 


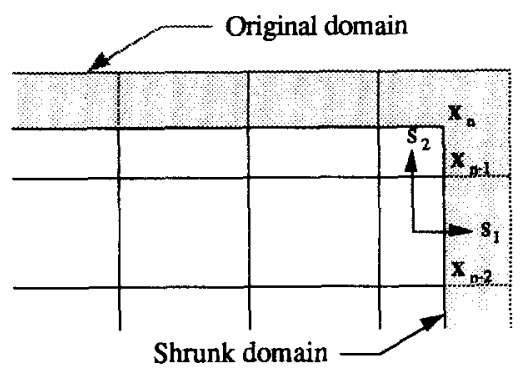

Fig. 15. Remapping based on the shrunk domain and extrapolation.

Another way to obtain the unique nodal value $q_{\mathrm{n}}$ from $\bar{q}$ is the weighted averaging scheme. This scheme gives $q_{\mathrm{n}}$ such that

$q_{\mathrm{n}}=\sum_{e=1}^{\mathrm{NAE}} \bar{q}_{e} W_{e} / \sum_{e=1}^{\mathrm{NAE}} W_{e}$,

where NAE is the total number of elements which surround the $n$-th nodal point and $W_{\mathrm{e}}$ is the weight of averaging scheme, which is taken to be the inverse of distance between the $n$-th nodal point and the center of the $e$-th element so that the $q$ may be the smoothest.

A significant disadvantage of this scheme is revealed in evaluation of $q_{\mathrm{n}}$ on the boundary. Since those nodal points are not fully surrounded by elements, $q_{\mathrm{n}}$ can not be obtained correctly. To overcome this, the following compensation scheme is introduced. We shall introduce the same averaging scheme to the nodal coordinate:

$x_{\mathrm{n}}=\sum_{e=1}^{\mathrm{NAE}} x_{e} W_{e} / \sum_{e=1}^{\mathrm{NAE}} W_{e}$

where $x_{\mathrm{n}}$ is considered to be "nodal" coordinate at which $q_{\mathrm{n}}$ is obtained, and $\boldsymbol{x}_{e}$ is the coordinate of the center of the $e$-th element. As shown in fig. 15, $x_{\mathrm{n}}$ construct a shrunk domain and the value at new location is obtained by (19) based on the shrunk domain. If the new location is outside of the shrunk domain, which is the shaded area in fig. 15, (19) works as extrapolation on the closest element in the shrunk domain. It is also noted that the weighted averaging scheme always gives underestimated values in the vicinity of a singular point, see [12] to avoid such a problem.

\section{Acknowledgements}

This work was done while the first author was at the University of Michigan. The research was partially supported by the Japan Research Institute, Limited.

\section{References}

[1] N. Kikuchi and T. Torigaki, Elastic-plastic impact/contact problems by adaptive finite element methods, Advances in Inelastic Analysis, ASME, AMD-vol. 88, PEDvol. 28 , (1987) 309-331.

[2] C.W. Gear and K.W. Tu, The effect of variable time step size on the stability of multistep methods, SIAM J. Numer. Anal. 11 (1974) 1025-1043.

[3] L.F. Shampine, Local error control in codes for ordinary differential equations, Appl. Math. Comp. 3 (1977).

[4] H.M. Hilber and T.J.R. Hughes, Collocation, dissipation and 'overshoot' for time integration schemes in structural dynamics, Earthquake Engineering and Structural Dynamics 6 (1978) 99-117.

[5] K.C. Park and P.G. Underwood, A variable central difference method for structural dynamic analysis - Part I. Theoretical aspects, Comput. Meth. Appl. Mech. Engrg. 22 (1980) 241-258.

[6] S.K. Lee, An integrated computer aided engineering approach for engine cylinder block analysis using HEXA8 finite elements, Ph. D. dissertation, Univ. of Michigan, Ann Arbor, MI (1989).

[7] P. Hartley, C.E.N. Sturgess and G.W. Rowe, Computer simulation of metalforming process (Elastic-plastic finite-element analysis of forging), Adv. Eng. Software 4, no. 1 (1982).

[8] K. Roll and T. Neitzert, On the application of different numerical methods to calculate cold forming precesses, in: J.F.T. Pittman et al., ed., Numerical Methods in Industrial Forming Processes (Pineridge Press, Swansea, U.K., 1982) pp. 97-107.

[9] C.J.M. Gelten and A.W.A. Konter, Application of meshrezoning in the updated Lagrangian method to metal forming analyses, in J.F.T. Pittman et al., ed., Numerical Methods in Industrial Forming Processes (Pineridge Press, Swansea, U.K., 1982) pp. 211-220

[10] G.L. Goudreau, Large scale computations, in: S. NematNasser, R.J. Asaro and G.A. Hegemier, eds. Theoretical Foundation for large-Scale Computations of Nonlinear Material Behavior (Martinus Nijhoff Publishers, Dordrecht, 1984) pp. 65-97.

[11] J.H. Cheng, Metal forming analysis based on large deformation elasto-plasticity, Ph. D. dissertation, Univ. of Michigan, Ann Arbor, MI (1985).

[12] T. Torigaki, Contact and impact problems using adaptive finite element methods, Ph. D. dissertation, Univ. of Michigan, Ann Arbor, MI (1989).

[13] R.E. Bank, Analysis of a local a posteriori error estimate for elliptic equations, in: I. Babuška et al., ed., Accuracy Estimates and Adaptive Refinements in Finite Element Computations, (John Wiley \& Sons, Chichester, 1986) pp. 119-128. 